\title{
Simple flow cytometric detection of haemozoin containing leukocytes and erythrocytes for research on diagnosis, immunology and drug sensitivity testing
}

Rosangela Frita ${ }^{1}$, Maria Rebelo ${ }^{1}$, Ana Pamplona ${ }^{1}$, Ana M Vigario ${ }^{1,2}$, Maria M Mota ${ }^{1}$, Martin P Grobusch ${ }^{3,4,5}$ and Thomas Hänscheid ${ }^{1,5^{*}}$

\begin{abstract}
Background: Malaria pigment (haemozoin, $\mathrm{Hz}$ ) has been the focus of diverse research efforts. However, identification of $\mathrm{Hz}$-containing leukocytes or parasitized erythrocytes is usually based on microscopy, with inherent limitations. Flow cytometric detection of depolarized Side-Scatter is more accurate and its adaptation to common bench top flow cytometers might allow several applications. These can range from the ex-vivo and in-vitro detection and functional analysis of Hz-containing leukocytes to the detection of parasitized Red-Blood-Cells (pRBCs) to assess antimalarial activity.

Methods: A standard benchtop flow cytometer was adapted to detect depolarized Side-Scatter. Synthetic and Plasmodium falciparum $\mathrm{Hz}$ were incubated with whole blood and PBMCs to detect $\mathrm{Hz}$-containing leukocytes and CD16 expression on monocytes. C5BL/6 mice were infected with Plasmodium berghei ANKA or P. berghei NK65 and $\mathrm{Hz}$-containing leukocytes were analysed using CD11b and Gr1 expression. Parasitized RBC from infected mice were identified using anti-Ter119 and SYBR green I and were analysed for depolarized Side Scatter. A highly depolarizing RBC population was monitored in an in-vitro culture incubated with chloroquine or quinine.
\end{abstract}

Results: A flow cytometer can be easily adapted to detect depolarized Side-Scatter and thus, intracellular Hz. The detection and counting of $\mathrm{Hz}$ containing leukocytes in fresh human or mouse blood, as well as in leukocytes from in-vitro experiments was rapid and easy. Analysis of CD14/CD16 and CD11b/Gr1 monocyte expression in human or mouse blood, in a mixed populations of $\mathrm{Hz}$-containing and non-containing monocytes, appears to show distinct patterns in both types of cells. Hz-containing pRBC and different maturation stages could be detected in blood from infected mice. The analysis of a highly depolarizing population that contained mature pRBC allowed to assess the effect of chloroquine and quinine after only 2 and 4 hours, respectively.

Conclusions: A simple modification of a flow cytometer allows for rapid and reliable detection and quantification of $\mathrm{Hz}$-containing leukocytes and the analysis of differential surface marker expression in the same sample of $\mathrm{Hz}$-containing versus non-Hz-containing leukocytes. Importantly, it distinguishes different maturation stages of parasitized RBC and may be the basis of a rapid no-added-reagent drug sensitivity assay.

\footnotetext{
* Correspondence: t.hanscheid@fm.ul.pt

${ }^{1}$ Instituto de Medicina Molecular, Faculdade de Medicina de Lisboa, Lisbon, Hospital Universitário de Santa Maria, Av. Prof. Egas Moniz, P-1649-028

Lisboa, Portugal

Full list of author information is available at the end of the article
} 


\section{Background}

The malaria pigment, or haemozoin $(\mathrm{Hz})$, is gaining increasing attention, as has been reviewed recently [1,2]: (i) $\mathrm{Hz}$ production is an important drug target, (ii) $\mathrm{Hz}$ appears to have immunomodulatory properties, (iii) detection of $\mathrm{Hz}$-containing leukocytes allows diagnosis of malaria, and (iv) Hz-containing leukocytes appear to be associated with disease severity. However, one important drawback in this area of research is the fact that counting of $\mathrm{Hz}$-containing leukocytes or the detection of $\mathrm{Hz}$ containing parasitized red-blood cells (pRBCs) is based on microscopy $[3,4]$. This is not only cumbersome, but introduces a significant statistical error if the number of $\mathrm{Hz}$-containing leukocytes is low [5]. An alternative to this is based on the detection of depolarized Side-Scatter by flow cytometry [6]. Hz is birefringent and as a consequence rotates the plane of polarized light, a process called depolarization. LASER light, commonly used in flow cytometers, produces polarized light. Thus, by placing a polarization filter orthogonally $\left(90^{\circ}\right.$ rotated) to the plane of the LASER light in front of a second SSC detector allows to detect depolarized light and consequently $\mathrm{Hz}$.

Depolarized SSC detection is incorporated into the Cell-Dyn ${ }^{\circledR}$ haematology analysers (Abbott, Santa Clara, CA, USA) to differentiate eosinophils from granulocytes. As a result of this, these instruments detect $\mathrm{Hz}$-containing leukocytes as well as $\mathrm{Hz}$ in parasitized red blood cells (pRBC) without need for modifications, $[7,8]$. Unfortunately, the software analysis and analysis algorithms of the analysers cannot be accessed without the intervention of the manufacturer and thus, studies depended on counting the events (cells) on the instrument's screen or the printout, with consequent data loss and no option for further analysis of the raw data [9]. However, if a simple modification of common flow cytometers created a reliable method for detection and analysis of $\mathrm{Hz}$ in leukocytes or pRBC it might open novel approaches for diagnostic applications and research.

For example, flow cytometric counting of Hz-containing leukocytes may be a better marker for disease severity than microscopy based counts $[5,10]$. Detection and functional analysis of $\mathrm{Hz}$-containing leukocytes may also help to elucidate further aspects on the immunomodulatory properties of Hz. In fact, most in-vitro studies use concentrations of $\mathrm{Hz}$ that maximize uptake by the majority of monocytes in a given cell population and compare them with a control population that was not exposed to $\mathrm{Hz}$ [11]. However, the situation may be different in vivo, where both populations coexist. For example, a recent large study, based on microscopy, reported that the median percentage of circulating Hz-containing monocytes was only $2-5 \%$ [3].
Interestingly, monocytes are a heterogeneous cell population, even in peripheral blood [12,13], mainly based on the CD14 and CD16 expression: classical "inflammatory" monocytes $\left(\mathrm{CD} 14^{+} / \mathrm{CD} 16^{-}\right)$, "intermediate" monocytes $\left(\mathrm{CD} 14^{+} / \mathrm{CD} 16^{+}\right)$and "resident, proinflammatory" monocytes (CD14 $\left.4^{\mathrm{dim}} / \mathrm{CD} 16^{+}\right)$. They also seem to differ functionally [14]. Recent research showed differences in the ratio of these populations in malarious children with different types of malaria [15], as well as differences in surface marker expression and parasite inhibitory action in acute uncomplicated malaria [16]. However, little is known whether $\mathrm{Hz}$ phagocytosis contributes to these findings in vivo. In fact, the interplay of $\mathrm{Hz}$-containing and non-Hz-containing subpopulations might produce different biological results in vivo, when compared to those in vitro models, where nearly all monocytes contain $\mathrm{Hz}$.

Another important area for flow cytometric detection of $\mathrm{Hz}$ could be the reliable detection of $\mathrm{pRBC}$ harbouring different $\mathrm{Hz}$ content. This may be useful for sensitivity testing and drug development, because the $\mathrm{Hz}$-content increases proportional to the maturation of the parasite [17].

This paper describes a simple way to modify a standard bench-top flow cytometer to allow depolarized Side-Scatter measurements. The method was then tested for its usefulness to detect $\mathrm{Hz}$-containing leukocytes and erythrocytes and examples for potential applications are presented.

\section{Methods}

\section{Flow cytometer modification (depolarized Side Scatter} detection)

The CyFlow ${ }^{\circledR}$ Blue (Partec, Münster, Germany) is a small, five parameter flow cytometer (Figure 1a) with blue laser $(488 \mathrm{~nm})$ excitation, and detectors for Forward Scatter (FSC), Side Scatter (SSC), green fluorescence (FL1), orange fluorescence (FL2) and red fluorescence (FL3) (Figure 1b). For this study the set-up was changed, mainly by creating two SSC detectors, using a $50 \% / 50 \%$ beam splitter. Then a polarization filter was placed orthogonally to the polarization plane of the laser light, in front of one of these SSC detectors which allowed the detection of depolarized Side-Scatter. This and other necessary changes are indicated in Figure 1c.

\section{Reagents}

All reagents were obtained from Sigma Aldrich (St Louis, Mo, USA), unless stated otherwise. Fluorescein isothiocyanate (FITC) or phycoerythrin (PE) labelled antibodies against surface antigens as well as isotype antibodies (CD14-FITC, CD16-PE, CD11b-FITC, Gr1-PE, TER119-PE, Fc-block) were purchased from 


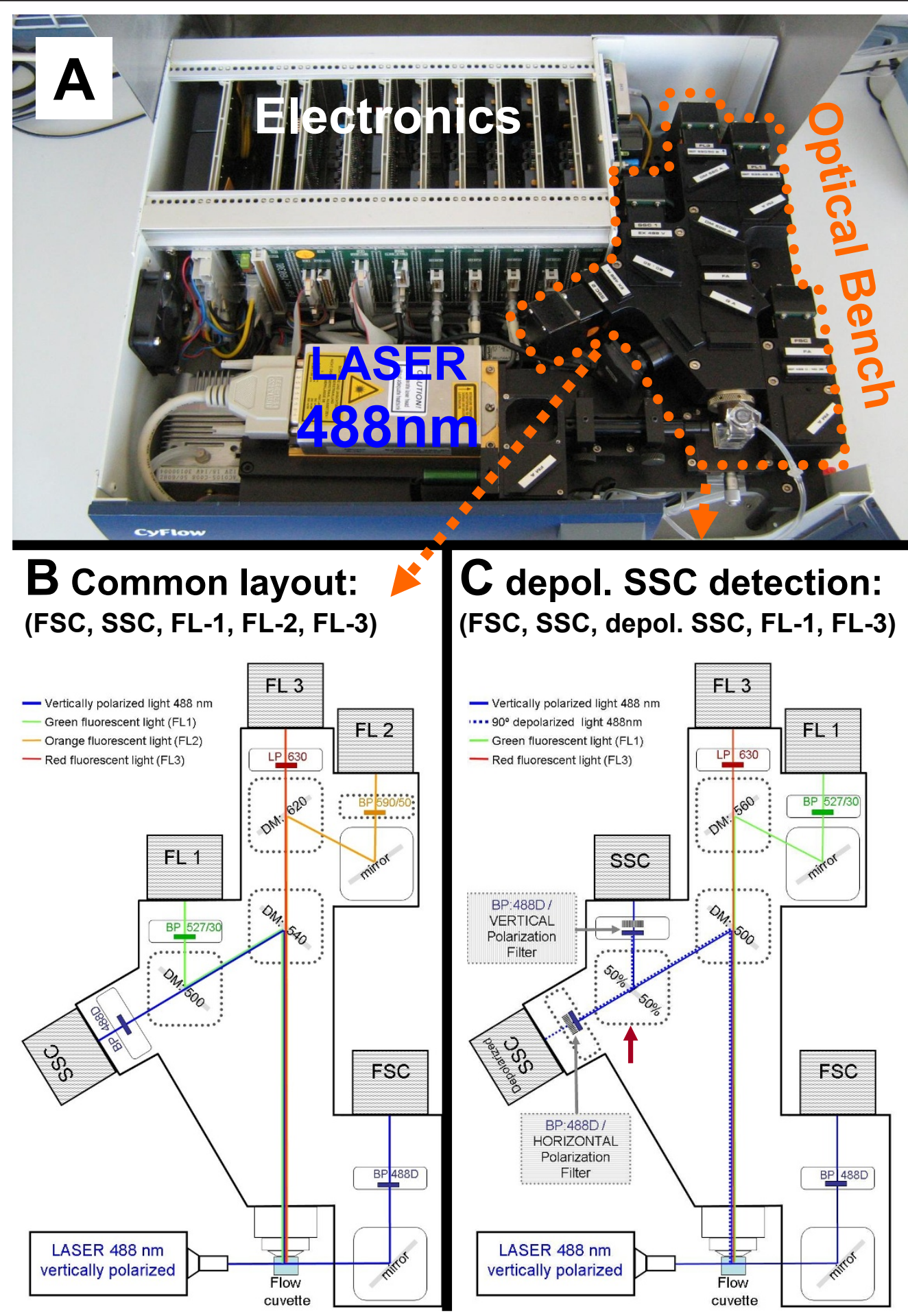

Figure 1 Alterations to the optical bench of a common bench-top flow cytometer which allows detection of depolarized side-scatter. a) The lid of the CyFLow ${ }^{\circledR}$ Blue flow cytometer can be easily removed and filters can be swapped by the operator. b) Light path in

conventional filter set-up for detection of Forward Scatter (FSC), Side-Scatter (SSC), green (FL-1), orange (FL-2) and red (FL-3) fluorescence. c) Filter set-up that allows detection of depolarized Side-Scatter instead of FL-2 detection. Squares with broken line indicate dichroic mirrors that need to be changed. Red error shows 50\%/50\% beam splitter. Other beam splitters which divert more light to the depolarized SSC are possible, such as $90 \% / 10 \%$ or even $95 \% / 5 \%$. DM = Dichroic Mirror; BP = Bandpass filter, LP = Longpass filter, numbers indicate wavelength in $\mathrm{nm}$. 
eBioscience (San Diego, USA). DNAse/RNAse free ultrapure water, Phosphate buffer saline (PBS), Fetal Bovine Serum (FBS), HEPES and RPMI 1640 were purchased from Gibco (Grand Island, NY). Ultrapure water was obtained with a Milli-Q purification system (Millipore, Madrid, Spain).

\section{Haemozoin preparation and quantification}

Synthetic haemozoin $(\mathrm{sHz})$ was prepared by dissolving $1.1 \mathrm{gr}$ of hemin chloride in $70 \mathrm{ml}$ of $0.4 \mathrm{M} \mathrm{NaOH}$ and $62 \mathrm{ml}$ of ultrapure water. The heme was then precipitated by the addition of $68 \mathrm{ml}$ of glacial acetic acid. The mixture was heated at $37^{\circ} \mathrm{C}$ over night to promote the formation of $\beta$-haematin. The following day, the formed crystalline synthetic haemozoin $(\mathrm{sHz})$ was washed 5 times in 5\% pyridine (in 0.02 M HEPES at $\mathrm{pH} 7.5$ ) and five times in ultrapure water. Finally, $\mathrm{sHz}$ was resuspended in PBS, quantified and stored at $4^{\circ} \mathrm{C}$.

Plasmodium. falciparum (strain 3D7) haemozoin (Pf-Hz) was purified from a synchronized culture at a parasitaemia of around 3-5\%. Infected erythrocytes were spun at $2000 \mathrm{rpm}$ for $10 \mathrm{~min}$ and resuspended for 10 min in a mixture of $40 \mathrm{ml}$ ultrapure water with $2 \mathrm{ml}$ of a $1 \%$ saponin in water. Cell lysates were then centrifuged at 13,200 rpm for $15 \mathrm{~min}$. The $\mathrm{Hz}$ pellets were washed $4 \mathrm{x}$ with PBS, resuspended in $1 \mathrm{ml}$ PBS, quantified and stored at $4^{\circ} \mathrm{C}$. Both types of $\mathrm{Hz}$ showed no DNA contamination and were negative for Mycoplasma.

The concentration of $\mathrm{Hz}$ was determined as haem content (haem-equivalent) after solubilization in $20 \mathrm{mM}$ $\mathrm{NaOH}$ for 1 hour at room temperature. The haem concentration was then determined by luminescence at $400 \mathrm{~nm}$ using the QuantiChrom Heme Assay Kit from BioAssay Systems (Hayward CA, USA).

\section{In vitro incubation of human whole blood with synthetic} haemozoin

Heparin-anticoagulated blood from healthy human donors was diluted 1:1 in RPMI 1640; and distributed into a 24 well plate. Then $\mathrm{sHz}$ was added at $0.01 ; 0.06$ and $0.12 \mu \mathrm{mol}$ haem-equivalent $/ \mathrm{ml}$. The plate was incubated for 7 hours at $37^{\circ} \mathrm{C}$ in $5 \% \mathrm{CO} 2$. Leucocytes were analysed in triplicates at time 0,4 and 7 hours.

In vitro incubation of human PBMCs with synthetic and $P$. falciparum haemozoin

Human PBMC were isolated from $40 \mathrm{ml}$ heparin-anticoagulated blood collected from healthy volunteers and after dilution 1:1 in RPMI 1640 and placed in a Ficoll gradient (Ficoll-Paque Plus, GE Healthcare, Uppsala, Sweden). Cells were centrifuged at $700 \mathrm{~g}$ for $20 \mathrm{~min}$ and the interface containing the PBMCs was collected. The PBMCs were washed, counted and resuspended at a concentration of $1 \times 10^{6} \mathrm{PBMC} / \mathrm{ml}$ in RPMI 1640 supplemented with $2 \mathrm{mM}$ L-glutamine, $0.05 \mathrm{mg} / \mathrm{ml}$ gentamicin and $10 \%$ foetal calf serum and distributed into a 24 well plate. Then $\mathrm{sHz}$ was added at 0.004 and 0.007 $\mu$ moles heme-equivalent $/ \mathrm{ml}$ and P.f.- $\mathrm{Hz}$ was added at 0.002 and $0.004 \mu \mathrm{mol}$ haem-equivalent $/ \mathrm{ml}$ in triplicates. Polystyrene latex beads $(0,1 \mu \mathrm{m})$ were diluted from the stock at $10 \%(\mathrm{vol} / \mathrm{vol})$ to a final concentration of $0.001 \%$ and used as control. The plate was incubated for 6 hours at $37^{\circ} \mathrm{C}$ in $5 \% \mathrm{CO} 2$.

\section{Flow cytometric analysis of human leukocytes}

EDTA anticoagulated blood was obtained from volunteers and patients for immediate analysis. One hundred $\mu \mathrm{l}$ of EDTA-anticoagulated blood was incubated for 20 minutes with anti-CD14 and anti-CD16. RBC were then lysed prior to analysis with Whole Blood Lysis Reagent (Partec, Münster, Germany) without further washing.

For the in vitro whole blood assay $50 \mu \mathrm{l}$ were incubated with anti-CD14 and anti-CD16 antibodies. RBC were lysed with BD FACS lysing solution (BD Biosciences, San Jose) for 5 minutes and washed before analysis. PBMCs were labelled as described for the whole blood assay, without erythrocyte lysis. All in-vitro samples were performed in triplicate. The gating strategy for monocytes and granulocytes and identification of $\mathrm{Hz}$-containing leukocytes is shown in Figure 2.

\section{Flow cytometric analysis of murine leukocytes}

Groups of five C57BL/6 mice (Charles River, Spain) were infected intraperitoneally with Plasmodium berghei ANKA (PbA) as model for experimental cerebral malaria or $P$. berghei NK65 (PbNK) as model for hyperparasitaemia. Uninfected and $P$. berghei NK65 infected mice were followed for 18 days. PbA-infected mice were sacrificed on day 5 when showing obvious signs of morbidity. Parasitaemia and flow cytometric analysis of blood was performed on days 3, 5, 12 and 18 post infection. At each time point approximately $25 \mu \mathrm{l}$ of blood was collected from a tail vein, incubated with Fc-block and then labelled with anti-CD11b and anti-Gr1. After washing the cells, RBC were lysed with $125 \mu$ l of BD FACS lysing solution (BD Biosciences, San Jose) for $5 \mathrm{~min}$. The cells were washed again, resuspended in FACS buffer and analysed by flow cytometry. Granulocytes and monocytes were analysed as described previously [18], in particular following a protocol that uses Side Scatter, CD11b and Gr1 expression [19] (see: additional file 1 - mouse blood gating strategy.pdf).

\section{Analysis of murine $\mathrm{Hz}$-containing RBC}

Mice (C57BL/6) (Charles River, Spain) were infected with P. berghei ANKA. Blood samples were collected when mice had a parasitaemia of approximately $5 \%$. RBC were incubated with Fc-block and then labelled 


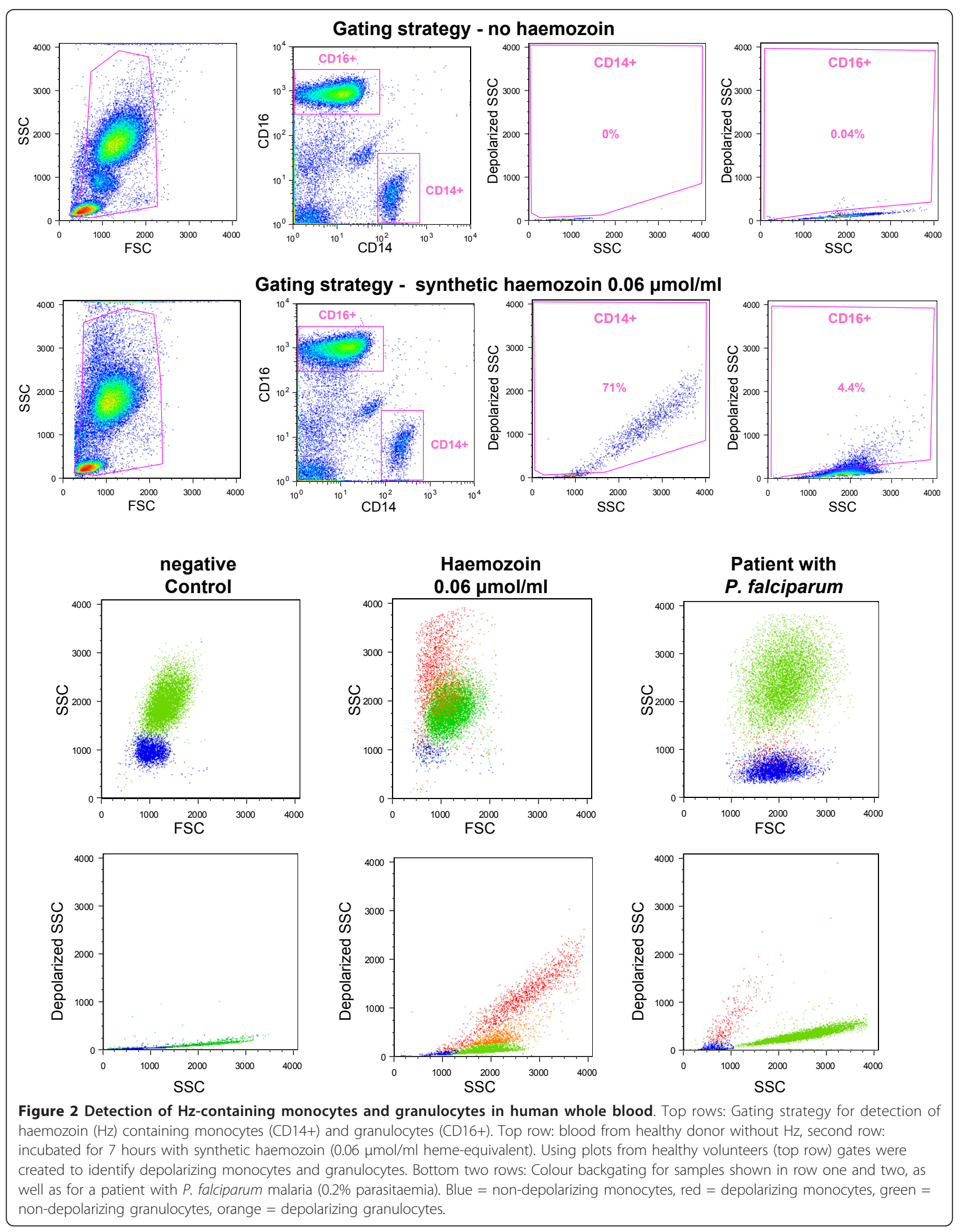


with anti-TER119. After washing the cells, SYBR green I (Invitrogen, Carlsbad, USA) was used to stain the DNA of intraerythrocytic parasites. Briefly, $2.5 \mu \mathrm{l}$ of the SYBR Green I solution at $10 \mathrm{x}$ was added to a blood suspension of approximately 400,000 cells/mL and incubated for 20 minutes, in the dark.

\section{Anti-malarial drug effect on $P$. berghei infected RBC in- vitro}

Blood from infected mice was diluted 1:50 in RPMI medium supplemented with $10 \%$ foetal calf serum, $1 \%$ non essential amino acids, $1 \%$ penicillin/streptomycin, $1 \%$ glutamine and $10 \mathrm{mM}$ Hepes, $\mathrm{pH}$ 7. The blood suspension was diluted 1:1 in complete RPMI medium and incubated with either chloroquine or quinine for 12 hours in a 24 well plate at $37^{\circ} \mathrm{C}$ in a $5 \% \mathrm{CO} 2$ atmosphere. Chloroquine diphosphate salt or quinine hydrochloride were dissolved in ultrapure water to prepare the stock solution. Final concentrations of $25 \mathrm{nM}$, $50 \mathrm{nM}$ and $100 \mathrm{nM}$ of chloroquine or $400 \mathrm{nM}$ and 800 $\mathrm{nM}$ of quinine were used. Flow cytometric measurements were done during every two hours by diluting 5 $\mu \mathrm{l}$ of the suspension present in the wells in $1 \mathrm{~mL}$ of FACS buffer. The effect of the anti-malarial drugs on the parasites was assessed by quantifying the percentage of highly depolarizing events (hdRBC).

All flow cytometry results were analysed using FlowJo software (version 9.0.2, Tree Star Inc., Oregon, USA).

The study was approved by the Ethical Committee of the Faculty of Medicine, University of Lisbon. All experiments involving animals were performed in compliance with the relevant laws and institutional guidelines.

\section{Results}

The Cyflow ${ }^{\circledR}$ instrument can be easily adapted to detect depolarized Side-Scatter (depol-SSC) by the operator, without any sophisticated tools or special knowledge. It is only necessary to open the instrument's cover, after releasing four screws, and change the respective filters and dichroic mirrors (Figure 1). This is a very simple procedure that lasts approximately 5 minutes. No special software is necessary and the same set-up (linear) as used for conventional SSC detection is sufficient, although the gain (voltage) of the detectors had to be adjusted.

\section{Detection of Hz-containing leukocytes in human blood} Using anti-CD14 and anti-CD16 it was possible to identify $\mathrm{Hz}$-containing monocytes and granulocytes in whole blood from healthy human volunteers incubated with $\mathrm{Hz}$ (Figure 2 and 3 ) as well as in a sample from a patient with malaria (Figure 2). Importantly, Hz-containing monocytes show much higher Side-Scatter than

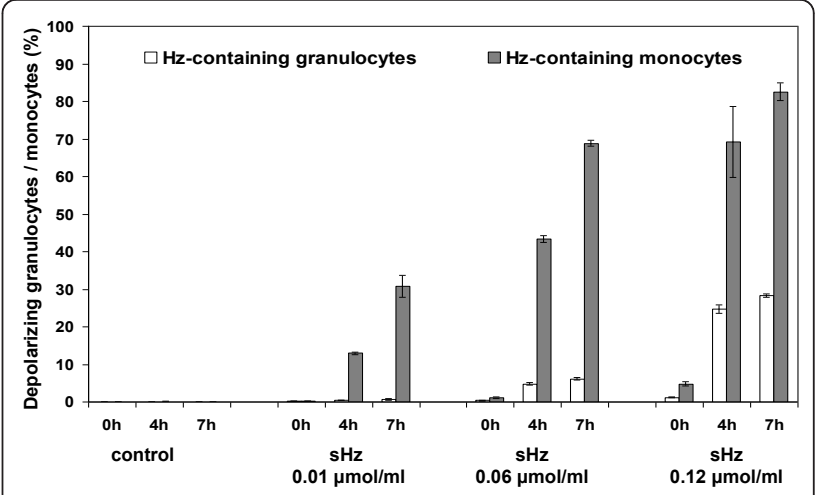

Figure 3 Phagocytosis of haemozoin in-vitro depends on dose and incubation time. Whole Blood from a healthy human volunteer was incubated with synthetic haemozoin $(\mathrm{sHz})$ at 0.01 , 0.06 and $0.12 \mu \mathrm{mol} / \mathrm{ml}$ of heme equivalent and with no $\mathrm{sHz}$ (control). The percentage of $\mathrm{Hz}$-containing granulocytes (open bars) and monocytes (grey bars) was determined at time zero, and after four and seven hours of incubation. Identification of leukocytes and depolarization as described in the text and shown in figure 2. Results are the mean values of triplicates ( \pm one SD).

normal monocytes, which overlap with the granulocyte population (Figure 2, bottom rows).

Incubation of whole blood from healthy volunteers with $\mathrm{sHz}$ shows that the percentage of $\mathrm{Hz}$-containing monocytes and granulocytes depends on the initial $\mathrm{Hz}$-dose and time of incubation (Figure 3).

In addition, different types of $\mathrm{Hz}$ cause distinct expression of CD16 on $\mathrm{Hz}$-containing monocytes, as compared with non-haemozoin containing monocytes in mixed populations (Figure 4, 5). Contrary to the synthetic $\mathrm{Hz}$ used in this study, P. falciparum derived $\mathrm{Hz}$ increases significantly CD16 expression on Hz-containing monocytes from healthy volunteers $(P<0.01)$; Figure 4 and 5). Interestingly, in a single case of $P$. falciparum malaria with $5.4 \% \mathrm{~Hz}$-containing monocytes, a substantial increase of CD16 expression was noticed and the majority of HZ-containing monocytes were clustered in the CD14+/CD16+ subpopulation (Figure 4, bottom row, right).

Altogether, the data clearly show that a simple alteration in a bench-top flow cytometer for detection of depolarized side-scatter allows quantification of $\mathrm{Hz}$-containing monocytes, which could be easily detected in a case of $P$. falciparum malaria.

\section{$\mathrm{Hz}$-containing murine leukocytes}

Using anti-CD11b and anti-Gr1 it was possible to identify $\mathrm{Hz}$-containing monocytes and granulocytes in mouse blood (Figure 6). Mice with $\mathrm{PbA}$ infection were sacrificed on day 5 because they developed symptoms compatible with experimental cerebral malaria. Overall, the results show that the percentage of $\mathrm{Hz}$-containing 


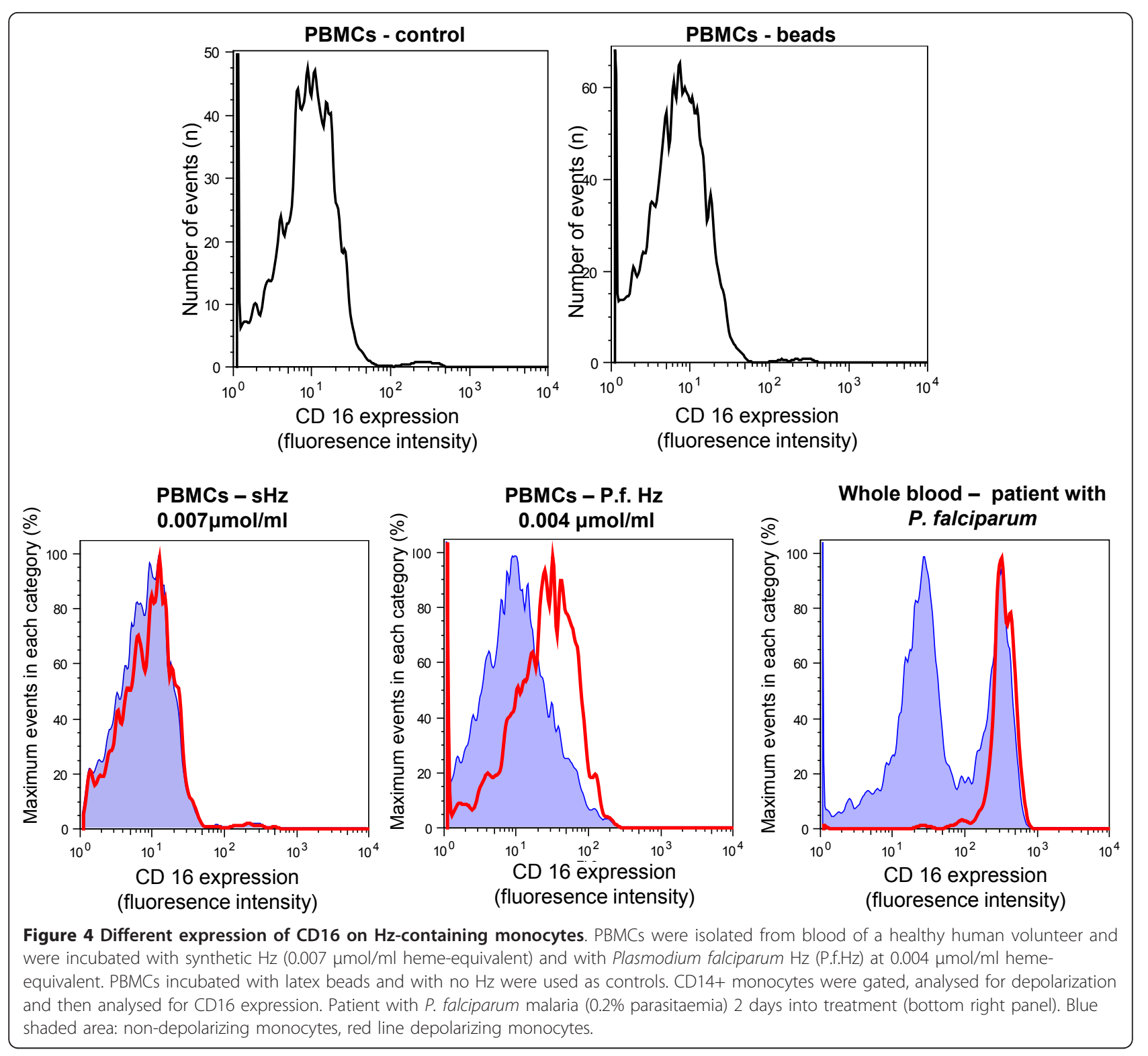

leukocytes increased when parasitaemia was higher (Figure 6). However, when mice infected with the two different parasite strains were compared when they had a similar parasitaemia, i.e. day 5 for $\mathrm{PbA}(5.7 \pm 3.1 \%)$ and day 12 for $\mathrm{PbNK}(7.7 \pm 1,3 \%)$, PbA infected mice had higher percentages of $\mathrm{Hz}$-containing monocytes than PbNK infected mice (Figure 6, top graph). Also, while the levels of parasitaemia of PbNK at day 18 of infection were significantly higher than the levels of parasitaemia of $\mathrm{PbA}$ at day 5 of infection $(28.5 \pm 12 \%$ and $5.7 \pm 3.1 \%$, respectively, $P<0,001)$ the percentages of $\mathrm{Hz}$-containing monocytes were almost the same $(13,3 \%$ and $13,0 \%$, respectively). Furthermore, a comparison of the degree of Gr1 expression (low, medium, high) on the Hz-containing monocytes, appeared to show differences in these two groups of mice at similar parasitaemia (Figure 6, bottom graph).

Interestingly, using $\mathrm{CD} 11 \mathrm{~b}$ and $\mathrm{F} 4 / 80$ showed that it appears possible to identify $\mathrm{Hz}$-containing tissue macrophages as shown in additional file 2 (Additional file 2 mouse spleen macrophages.pdf).

Altogether, using two distinct rodent models of infection, $\mathrm{Hz}$-containing monocytes and granulocytes can also be detected in this flow cytometer. Most importantly, the data suggest that the percentage and type of $\mathrm{Hz}$-containing monocytes may also be a associated with disease severity.

\section{Detection of $\mathrm{Hz}$ in murine pRBCs}

Plasmodium accumulates $\mathrm{Hz}$ throughout its development stage inside RBCs. The detection of depolarization 


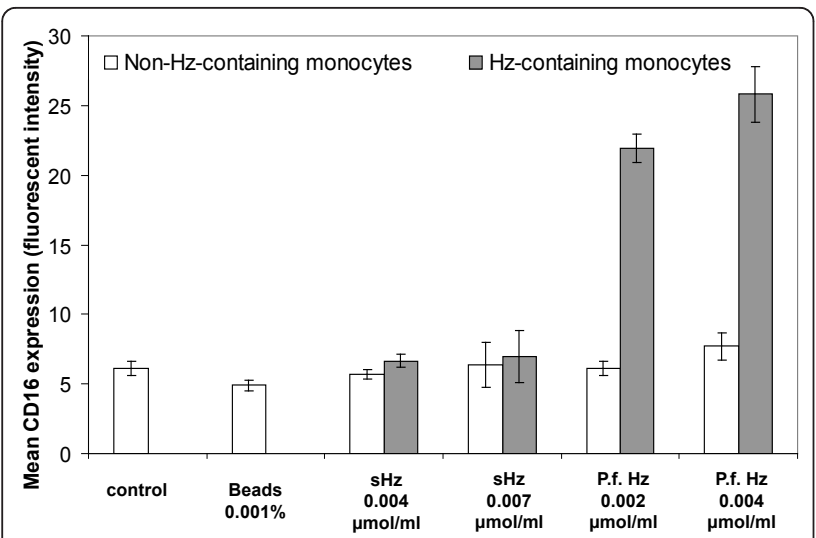

Figure 5 CD16 expression on monocytes fed with different types of haemozoin in-vitro. PBMCs were isolated from blood of a healthy human volunteer and were incubated with synthetic $\mathrm{Hz}$ $(\mathrm{sHz})$ at 0.004 and $0.007 \mu \mathrm{moles} / \mathrm{ml}$ heme equivalent and with Plasmodium falciparum $\mathrm{Hz}$ (P.f.Hz) at 0.002 and $0.004 \mu \mathrm{mol} / \mathrm{ml}$ heme equivalent. PBMCs incubated with latex beads and with no $\mathrm{Hz}$ were used as controls. Cells were incubated for 6 hours and then labelled with anti-CD14 and anti-CD16. The Geometric mean values for expression of CD16 were calculated for non- $\mathrm{Hz}$ containing monocytes (open bars) and $\mathrm{Hz}$-containing monocytes (grey bars). Differences between $\mathrm{sHZ}$ and P.f. were significant $(P<0.01)$. Shown are values of triplicates ( \pm one SD). Controls and beads had no depolarizing populations.

was evaluated to determine whether this measurement could be used to detect pRBCs and most importantly to distinguish different pRBC stages. In whole blood from mice infected with $P$. berghei a population of depolarizing events is observed (Figure 7). More than $97 \%$ of these events were positive for the RBC marker TER119 (red population, Figure 7E). SYBR green I staining was positive in this population, indicating that the depolarizing erythrocytes contained DNA, and thus were parasitized RBC (pRBC) (Figure 8). Further analysis revealed that events with a higher degree of depolarization also showed a higher degree of SYBR green fluorescence (Figure 8), with several distinct peaks, likely representing different maturation stages of the parasite (ring-forms, early and late trophozoites and schizonts).

\section{Detection of $\mathrm{Hz}$ in parasitized murine $\mathrm{RBC}$ to assess drug effects}

Based on the above findings (Figure 8 , gate A), which contained the pRBC with the highest degree of depolarization (hdRBC) and as such contains the most mature parasites, the flow cytometric method was investigated to see if it could be used to rapidly assess drug effects. Thus, the percentage of this hdRBC population was determined over a 12-hour period in an in-vitro culture of $P$. berghei pRBC. The results clearly show that the inhibitory effect of chloroquine and quinine is detected in a dose dependent manner throughout time (Figure 9).
Importantly, inhibitory effects were discernable after only two hours for chloroquine and four hours for quinine.

\section{Discussion}

\section{Modification of flow cytometers}

This study shows that a simple modification of a common flow cytometer for the detection of depolarized Side-Scatter, allows the detection of $\mathrm{Hz}$-containing leukocytes and erythrocytes. In fact, a study using a MoFlo ${ }^{\circledR}$ high speed cell sorter (Beckman Coulter, Inc, Fullerton, CA), showed that depolarizing leukocytes did contain $\mathrm{Hz}$ [20]. However, $\mathrm{MoFlo}^{\circledR}$ sorters are expensive and sophisticated instruments, and by far not as commonly available as flow cytometers, which are even standard equipment in most research centres in malaria endemic regions nowadays. Nonetheless, the widespread incorporation of this method in common flow cytometers of major manufacturers has somehow been hampered by the fact that the company of the CellDyn ${ }^{\circledR}$ instruments held the patent of this method (US patent 5017497) [21]. However, this patent was filed in 1989 and published in 1991, and as such, according to US legislation [22], it expired in March 2009. The necessary filters and dichroic mirrors (Figure 1c) used to change the CyFlow ${ }^{\circledR}$ cytometer were obtained for less than $€ 2.000$. No special software is necessary and the settings resemble those of the conventional SSC detection, albeit with an increased gain (voltage) of the detector. For the set-up in this study a $50 \% / 50 \%$ beam splitter used to divert the respective light to the depolarized SSC and SSC detector (Figure 1, red error). Using a $90 \% / 10 \%$ or even $95 \% / 5 \%$ beam splitter may increase the signal of the depolarized SCC, without decreasing the quality of the SSC signal.

Any flow cytometer can be modified as described here. However, it should be noted that to modify some flow cytometers, which use fibre optic cables for light collection, for example, the newer Becton Dickinson instruments (Franklin Lakes, NJ, USA), it is necessary to confirm that these cables maintain polarization of the light.

\section{Detection and counting of $\mathrm{Hz}$-containing leukocytes}

$\mathrm{Hz}$-containing monocytes and granulocytes could be rapidly determined in fresh human or mouse blood as well as in in-vitro phagocytosis experiments involving whole blood or PBMCs. As has been pointed out before, previous studies on the immunological effects of $\mathrm{Hz}$ invitro used diverse quantities of $\mathrm{Hz}$, different incubation times, as well as different types of phagocytic cells $[1,2]$. Few studies quantified the uptake of $\mathrm{Hz}$ by monocytes, although some used a luminescence assay after lysing the monocytes and reported $\mathrm{Hz}$ uptake as $\mathrm{Hz}$ - 

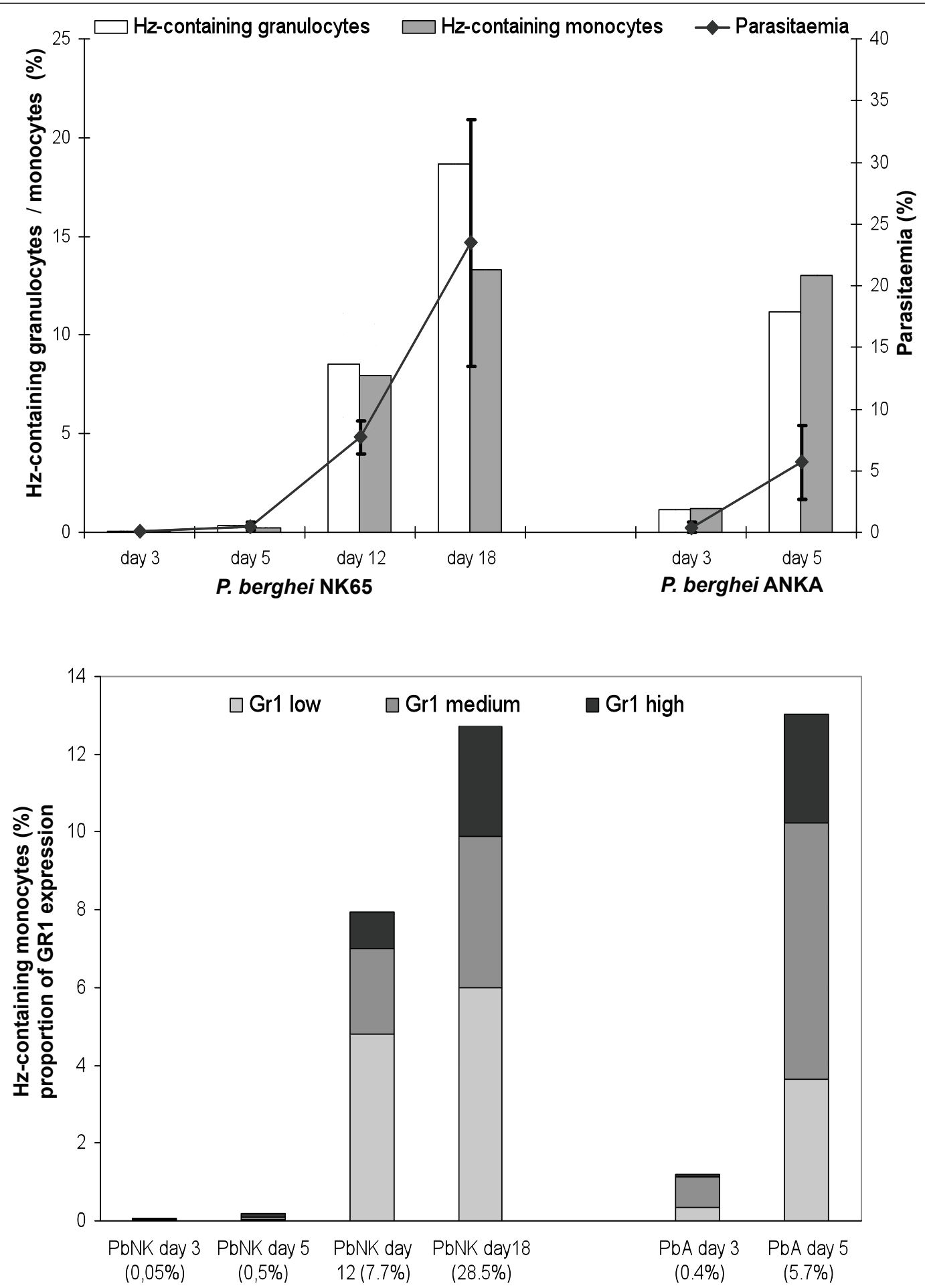

Figure 6 Haemozoin containing leukocytes in two different mouse models of malaria. Groups with five C57BL/6 mice where infected with P. berghei ANKA (PbA) or with P. berghei NK65 (PbNK). Blood was drawn on day 3, 512 and 18 and analysed after labelling with with anti-CD11b and Gr1. Analyses and gating strategy is described in the text (additional file 1). Percentage of $\mathrm{Hz}$-containing monocytes and granulocytes (top graph) as well as parasitaemia are mean values per group ( \pm one SD). Open bars $=\mathrm{Hz}$-containing granulocytes; grey bars $=\mathrm{Hz}$-containing monocytes. Uninfected controls had no Hz-containing leukocytes and are not shown. Bottom graph shows proportion of different Gr1 expression (high = black, medium = grey; and low = light grey) of Hz-containing monocytes, shown in top figure. Percentages on $x$-axis represent mean parasitemia. Mice with PbANKA infection were sacrificed on day 5. 


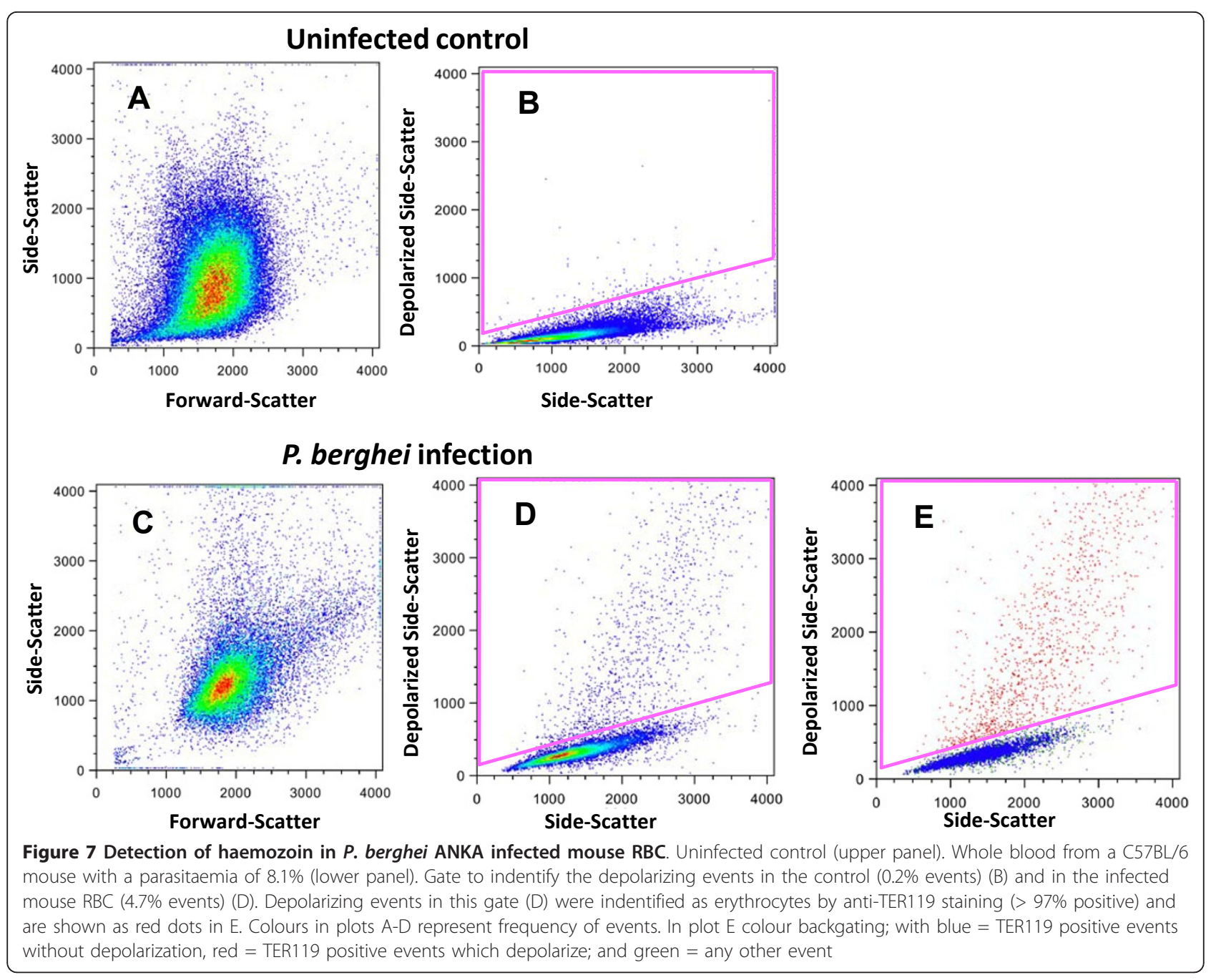

equivalents found in around 8-10 trophozoites per monocyte [23]. However, even less studies mention the percentage of Hz-containing monocytes. In one of these studies confocal microscopy was used and showed that $75 \%( \pm 31 \%)$ of human PBMCs contained $\mathrm{Hz}$ after only 180 min, albeit only based on counting a total of $\geq 400$ cells [11]. Contrary to this, the presented method allows the analysis of ten-thousands of cells to determine rapidly adequate doses of $\mathrm{Hz}$ and required incubation times to get the desired percentage of $\mathrm{Hz}$ containing leukocytes (Figure 3).

Furthermore, it allows to investigate $\mathrm{Hz}$-containing monocytes from malarious patients who usually have only a few percent of these cells in circulation [3]. For example, considering that in common protocols for the analysis of whole blood, $100 \mu \mathrm{l}$ are labelled, and further assuming 1,000 monocytes/ $\mu \mathrm{l}$ with only $5 \%$ of these containing $\mathrm{Hz}$, a total of $5000 \mathrm{~Hz}$-containing cells could be easily analysed using the flow cytometric method proposed here. As previously reported [5,10], this method also allows to re-address the question if the number of $\mathrm{Hz}$-containing leukocytes is associated with disease severity or certain types of malaria.

In some studies, however, the identification of monocytes was based on the typical monocyte gate in the Forward Scatter (FSC)/SideScatter (SSC) plot $[16,20]$. One problem with this is that monocytes containing several or larger $\mathrm{Hz}$ crystals might thus be excluded from analysis as they have higher SSC and appear superimposed on the granulocyte population (Figure 2), with a possible impact on final results.

\section{Single cell analysis for studies of $\mathrm{Hz}$-containing leukocytes}

This may open the possibility to investigate functional differences of $\mathrm{Hz}$-containing and non-containing leukocytes, especially in monocyte subsets found in fresh human blood from malarious patients or in mouse models of malaria. For example, recent papers reported differences in surface markers on CD14/CD16 monocyte 


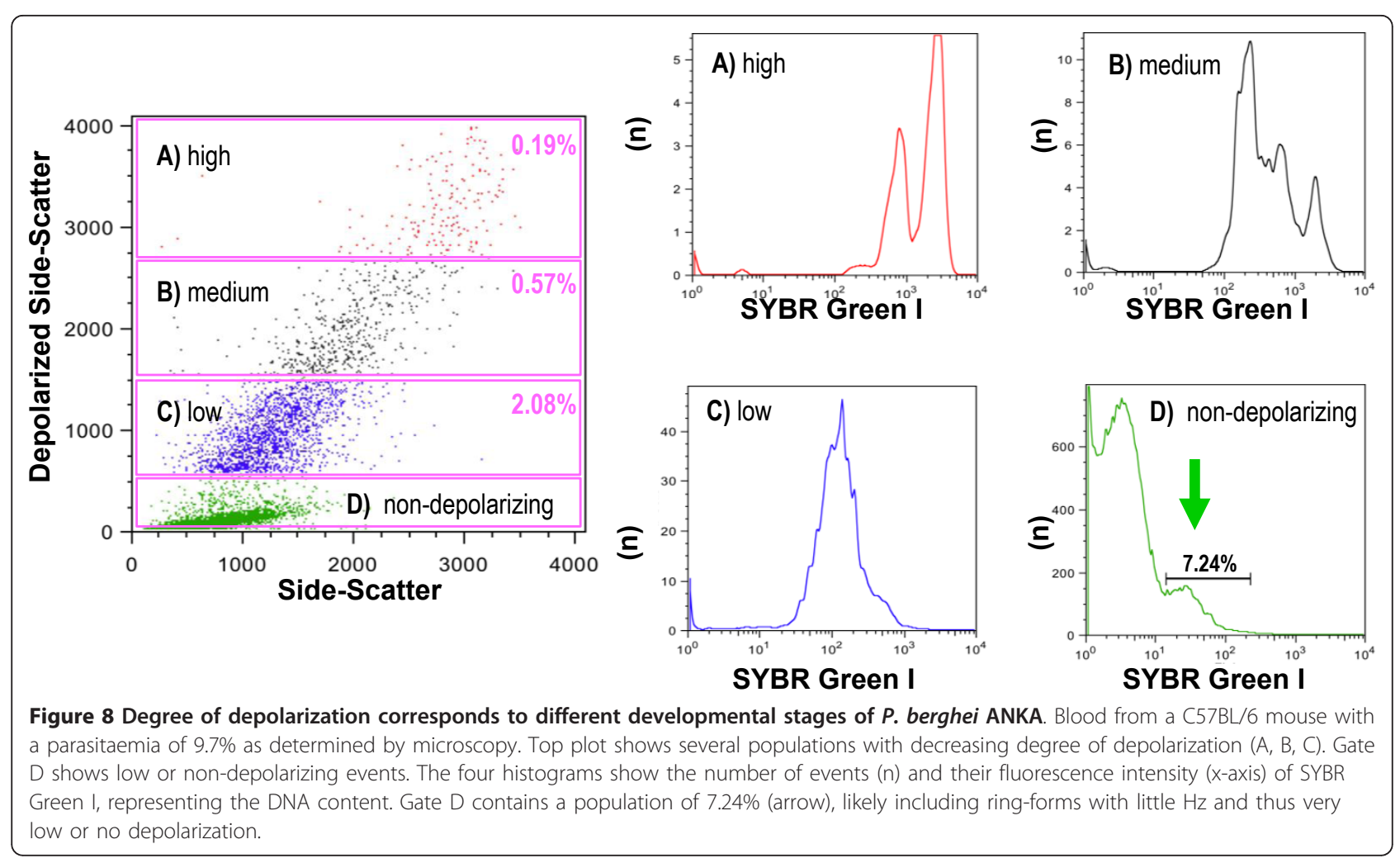

subsets in malarious patients $[15,16]$, or in peripheral blood dendritic cells in children [24]. Measurement of depolarized Side-Scatter and, thus, identification of $\mathrm{Hz}-$ containing cells might allow to establish if these cells may have an imported role in the reported results. It would also allow the possibility to study functionally leukocytes on single cell level in-vitro, including surface expression or intracellular cytokines, comparing $\mathrm{Hz}$ with non- $\mathrm{Hz}$ containing leukocytes in the same cell population. In fact, differences in CD16 expression in a mixed population of $\mathrm{Hz}$-containing and non-containing cells were observed (Figures 4 and 5), a finding that differs from a previous report that found no difference in CD16 expression in an in-vitro model [25]. Despite recent reports of differences between human and mouse monocytes [26], the current method may also allow to investigate the effects of $\mathrm{Hz}$ on phagocytic cells in fresh blood using different mouse models (Figure 6).

First results (additional file 2) indicate that it appears possible to detect tissue macrophages. This may allow to compare $\mathrm{Hz}$-containing and non-Hz-containing cells from tissues such as spleen, liver or bone marrow which may help to elucidate recent findings, for example, the association of $\mathrm{Hz}$ with severe anaemia $[27,28]$. However, the reliable identification of tissue macrophages may require more than the two antibodies used in this study. In this case, instruments that have several light sources are necessary.

\section{Detection of $\mathrm{pRBC}$ and sensitivity testing of antimalarious drugs}

Flow cytometric analysis of depolarized Side-Scatter allows to easily detect pRBCs (Figure 7) and distinguish different maturation stages of the parasite (Figure 8 ). Both findings might serve as the basis for a novel drug sensitivity assay. In fact, the idea to use $\mathrm{Hz}$ as a maturation indicator is not new and was tried in a simple visual agglutination test in the 1980s [17]. Apparently, this method did not produce very reliable results, mainly due to leukocyte interference [29]. However, these limitations could be overcome by single-cell flow cytometric analysis.

Currently several drug sensitivity assays for P. falciparum exist and have been reviewed elsewhere [29,30]. Nonetheless, all of these assays have some disadvantages, including long turn-around times or the need for additional and often expensive reagents. Interestingly, a recent report concluded that the reliability of some of these assays may depend on the mode of action of the tested drugs [31]. Flow cytometry appears to be a reliable technique to assess parasite maturation and sensitivity to antimalarial drugs. However, protocols reported so far require additional reagents, usually to stain DNA and/or RNA and may even require flow cytometers with more than one light source [32,33].

The presented method is based on the detection of $\mathrm{Hz}$, without the need of any further reagents. Determining the percentage of highly depolarizing RBC (hdRBC) 


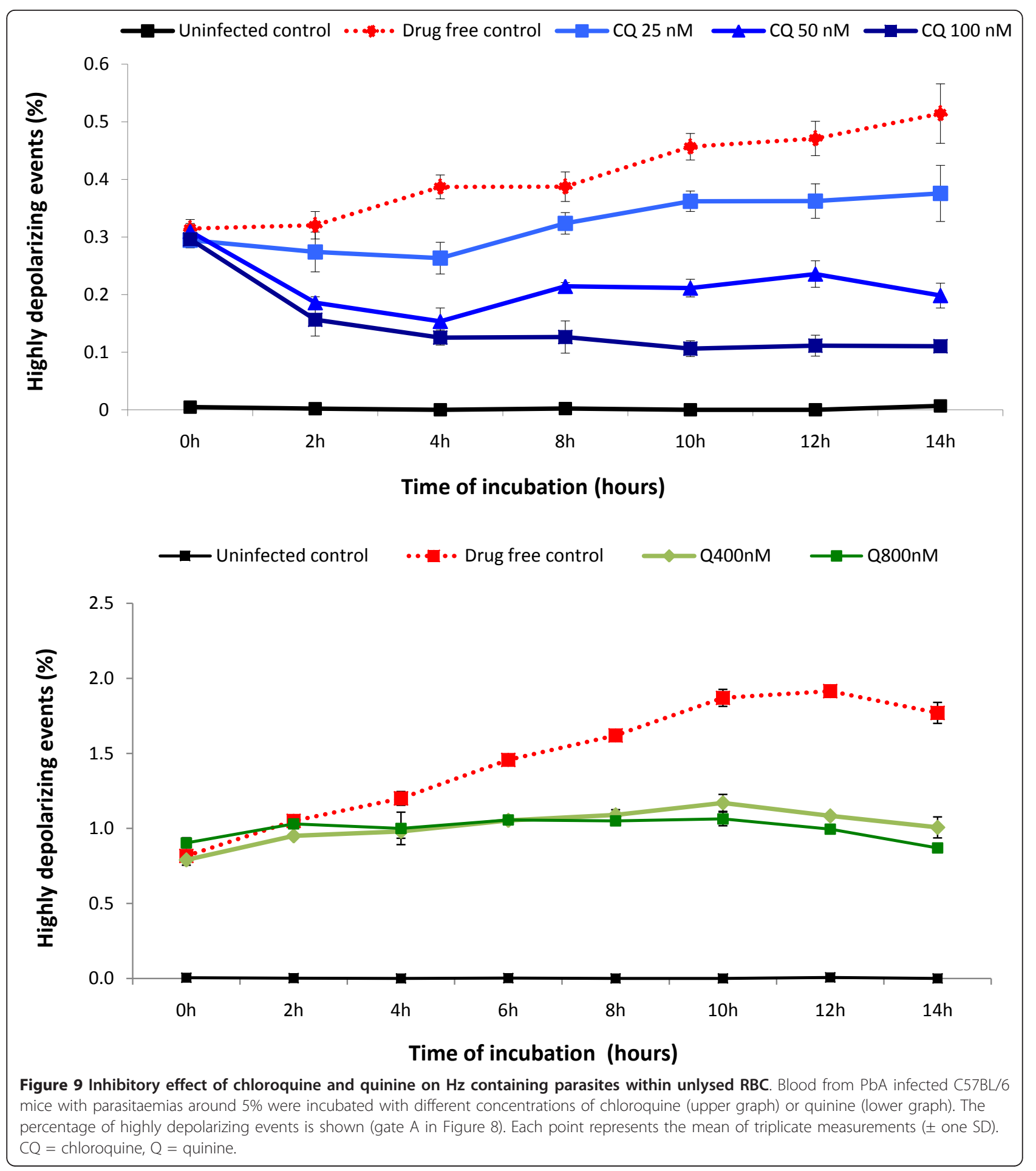

present in blood samples from infected mice, it was possible to detect the inhibitory effect of chloroquine (CQ) and quinine $(\mathrm{Q})$ after only a few hours of in-vitro culture (Figure 9). In this study an experimental model using C57BL/6 mice infected with $P$. berghei was chosen to assure that only maturation of parasites was measured, because $P$. berghei does not replicate in invitro cultures [34]. However, although the preliminary results seem promising, further work with different drugs and, most importantly, P. falciparum cultures are necessary to evaluate the usefulness of this method as a novel sensitivity assay. 


\section{Conclusions}

A simple modification of a flow cytometer allows the reliable detection of $\mathrm{Hz}$ containing leukocytes and pRBCs. This method facilitates the rapid and reliable detection and counting of $\mathrm{Hz}$-containing leukocytes in human or animal blood. It also allows single cell analysis of $\mathrm{Hz}$-containing versus non-Hz-containing leukocytes in the same sample, including functional analysis, both in-vitro experiments or using fresh blood. Finally, it detects different maturation stages of parasitized RBC and may be the basis of a rapid no-added-reagent drug sensitivity assay.

\section{Additional material}

Additional file 1: Description of flow cytometric gating strategy to indentify $\mathrm{Hz}$-containing mouse granulocytes and monocytes.

Additional file 2: Detection of $\mathrm{Hz}$-containing mouse spleen macrophages.

\section{Acknowledgements and Funding}

This work was funded by the Fundação para a Ciência e Tecnologia (PIC/IC) 83214/2007) and the Fundação Luso Americana (FLAD-LACR grant: B-A.V109-09/07). Rosangela Frita was supported by the Fundação Glaxo Smith Kline Research with a grant for a doctorate in Infectious Pathology.

\section{Author details}

${ }^{1}$ Instituto de Medicina Molecular, Faculdade de Medicina de Lisboa, Lisbon, Hospital Universitário de Santa Maria, Av. Prof. Egas Moniz, P-1649-028 Lisboa, Portugal. ${ }^{2}$ University of Madeira, Funchal, Portugal. ${ }^{3}$ Infectious Diseases, Tropical Medicine and AIDS, Division of Internal Medicine, Academic Medical Centre, University of Amsterdam, The Netherlands. ${ }^{4}$ Institute of Tropical Medicine, University of Tübingen, Germany. ${ }^{5}$ Medical Research Unit, Hôpital Albert Schweitzer, Lambaréné, Gabon.

\section{Authors' contributions}

RF and MR contributed equally to this paper

RF carried out the human and mouse leukocyte studies. MR realized the studies on parasitized RBC and anti-malarious drug effects. AP, AMV and MMM participated in setting up the mouse models and analysing the results. MMM and $\mathrm{TH}$ conceived the study, participated in its design and coordination. MPG contributed to the design of the study and the draft of the manuscript. All authors have read the manuscript and approved it.

\section{Competing interests}

The authors declare that they have no competing interests.

Received: 1 February 2011 Accepted: 31 March 2011

Published: 31 March 2011

\section{References}

1. Hänscheid T, Egan TJ, Grobusch MP: Hemozoin: from melatonin pigment to drug target, diagnostic tool, and immune modulator. Lancet Infect Dis 2007, 7:675-685.

2. Shio MT, Kassa FA, Bellemare MJ, Olivier M: Innate inflammatory response to the malarial pigment hemozoin. Microbes Infect 2010, 12:889-899.

3. Kremsner PG, Valim C, Missinou MA, Olola C, Krishna S, Issifou S, Kombila M, Bwanaisa L, Mithwani S, Newton CR, Agbenyega T, Pinder M, Bojang K, Wypij D, Taylor T: Prognostic value of circulating pigmented cells in african children with malaria. J Infect Dis 2009, 199:142-150.
4. Lawrence C, Olson JA: Birefringent hemozoin identifies malaria. Am J Clin Pathol 1986, 86:360-363.

5. Hanscheid T, Frita R, Langin M, Kremsner P, Grobusch M: Is flow cytometry better in counting malaria pigment-containing leukocytes compared to microscopy? Malar J 2009, 8:255.

6. de Grooth BG, Terstappen LW, Puppels GJ, Greve J: Light-scattering polarization measurements as a new parameter in flow cytometry. Cytometry 1987, 8:539-544.

7. Mendelow BV, Lyons C, Nhlangothi P, Tana M, Munster M, Wypkema E, Liebowitz L, Marshall L, Scott S, Coetzer TL: Automated malaria detection by depolarization of laser light. Br J Haematol 1999, 104:499-503.

8. Suh I, Kim H, Kim J, Lee S, An S, Kim W, Lim C: Evaluation of the Abbott Cell-Dyn 4000 hematology analyzer for detection and therapeutic monitoring of Plasmodium vivax in the Republic of Korea. Trop Med Int Health 2003, 8:1074-1081.

9. Hanscheid T, Langin M, Lell B, Potschke M, Oyakhirome S, Kremsner P, Grobusch M: Full blood count and haemozoin-containing leukocytes in children with malaria: diagnostic value and association with disease severity. Malar J 2008, 7:109.

10. Shankar AH, Fawzi WW: Moving toward hematological predictors of disease severity in malaria: going with the flow. Am J Hematol 2010, 85:225-226.

11. Schwarzer E, Bellomo G, Giribaldi G, Ulliers D, Arese P: Phagocytosis of malarial pigment hemozoin by human monocytes: a confocal microscopy study. Parasitology 2001, 123:125-131.

12. Strauss-Ayali D, Conrad SM, Mosser DM: Monocyte subpopulations and their differentiation patterns during infection. J Leukoc Biol 2007, 82:244-252.

13. Tacke F, Randolph GJ: Migratory fate and differentiation of blood monocyte subsets. Immunobiology 2006, 211:609-618.

14. Skrzeczyńska-Moncznik J, Bzowska M, Loseke S, Grage-Griebenow E, Zembala M, Pryjma J: Peripheral blood CD14high CD16+ monocytes are main producers of IL-10. Scand J Immunol 2008, 67:152-159.

15. Ogonda LA, Orago ASS, Otieno MF, Adhiambo C, Otieno W, Stoute JA: The levels of CD16/Fc\{gamma\} receptor IIIA on CD14+ CD16+ monocytes are higher in children with severe Plasmodium falciparum anemia than in children with cerebral or uncomplicated Malaria. Infect Immun 2010, 78:2173-2181.

16. Chimma P, Roussilhon C, Sratongno P, Ruangveerayuth $R$, Pattanapanyasat K, Pérignon JL, Roberts DJ, Druilhe P: A distinct peripheral blood monocyte phenotype is associated with parasite inhibitory activity in acute uncomplicated Plasmodium falciparum malaria. PLOS Pathog 2009, 5:e1000631.

17. Rieckmann $\mathrm{KH}$ : Visual in-vitro test for determining the drug sensitivity of Plasmodium falciparum. Lancet 1982, 1:1333-1335.

18. Sunderkötter C, Nikolic T, Dillon MJ, van Rooijen N, Stehling M, Drevets DA, Leenen PJM: Subpopulations of mouse blood monocytes differ in maturation stage and inflammatory response. J Immunol 2004, 172:4410-4417.

19. Aging study: Peripheral blood leukocytes (PBL profiles) in 32 inbred strains of mice. [http://phenome.jax.org/db/q?rtn=projects/ docstatic\&doc=Petkova1/Petkova1_Protocol].

20. Kramer B, Grobusch M, Suttorp N, Neukammer J, Rinneberg H: Relative frequency of malaria pigment-carrying monocytes of nonimmune and semi-immune patients from flow cytometric depolarized side scatter. Cytometry 2001, 45:133-140.

21. Particle discriminator and method, United States Patent 5017497. [http:// www.freepatentsonline.com/5017497.html].

22. How to Calculate Standard Patent Expiry Dates and Data Exclusivity in Key Territories. [http://www.genericsweb.com/ How_to_Calculate_Standard_Patent_Expiry_Dates_and_Data_Exclusivity. pdf].

23. Giribaldi G, Prato M, Ulliers D, Gallo V, Schwarzer E, Akide-Ndunge OB, Valente E, Saviozzi S, Calogero RA, Arese P: Involvement of inflammatory chemokines in survival of human monocytes fed with malarial pigment. Infect Immun 2010, 78:4912-4921.

24. Urban BC, Mwangi T, Ross A, Kinyanjui S, Mosobo M, Kai O, Lowe B, Marsh K, Roberts DJ: Peripheral blood dendritic cells in children with acute Plasmodium falciparum malaria. Blood 2001, 98:2859-2861. 
25. Schwarzer E, Alessio M, Ulliers D, Arese P: Phagocytosis of the malarial pigment, hemozoin, impairs expression of major histocompatibility complex class II antigen, CD54, and CD11c in human monocytes. Infect Immun 1998, 66:1601-1606.

26. Ingersoll MA, Spanbroek R, Lottaz C, Gautier EL, Frankenberger M, Hoffmann R, Lang R, Haniffa M, Collin M, Tacke F, Habenicht AJ, ZieglerHeitbrock L, Randolph GJ: Comparison of gene expression profiles between human and mouse monocyte subsets. Blood 2010, 115:e10-19.

27. Davenport GC, Ouma C, Hittner JB, Were T, Ouma Y, Ong'echa JM, Perkins DJ: Hematological predictors of increased severe anemia in Kenyan children coinfected with Plasmodium falciparum and HIV-1. Am J Hematol 2010, 85:227-233.

28. Lamikanra AA, Theron M, Kooij TWA, Roberts DJ: Hemozoin (malarial pigment) directly promotes apoptosis of erythroid precursors. PLOS ONE 2009, 4:e8446.

29. Basco L: Field application of in vitro assays for the sensitivity of human malaria parasites to antimalarial drugs Geneva: World Health Organization; 2007.

30. Noedl H, Wongsrichanalai C, Wernsdorfer WH: Malaria drug-sensitivity testing: new assays, new perspectives. Trends Parasitol 2003, 19:175-181.

31. Wein S, Maynadier M, Tran Van Ba C, Cerdan R, Peyrottes S, Fraisse L, Vial H: Reliability of antimalarial sensitivity tests depends on drug mechanisms of action. J Clin Microbiol 2010, 48:1651-1660.

32. Grimberg BT, Jaworska MM, Hough LB, Zimmerman PA, Phillips JG: Addressing the malaria drug resistance challenge using flow cytometry to discover new antimalarials. Bioorg Med Chem Lett 2009, 19:5452-5457.

33. Izumiyama S, Omura M, Takasaki T, Ohmae H, Asahi H: Plasmodium falciparum: Development and validation of a measure of intraerythrocytic growth using SYBR Green I in a flow cytometer. Exp Parasitol 2009, 121:144-150.

34. Janse CJ, Waters AP: Plasmodium berghei: The application of cultivation and purification techniques to molecular studies of malaria parasites. Parasitol Today 1995, 11:138-143.

doi:10.1186/1475-2875-10-74

Cite this article as: Frita et al: Simple flow cytometric detection of haemozoin containing leukocytes and erythrocytes for research on diagnosis, immunology and drug sensitivity testing. Malaria Journal 2011 $10: 74$.

\section{Submit your next manuscript to BioMed Central and take full advantage of:}

- Convenient online submission

- Thorough peer review

- No space constraints or color figure charges

- Immediate publication on acceptance

- Inclusion in PubMed, CAS, Scopus and Google Scholar

- Research which is freely available for redistribution

Submit your manuscript at www.biomedcentral.com/submit 